\title{
Discrete interferences optimum beamformer in correlated signal and interfering noise
}

\author{
Satyanand Singh ${ }^{1}$, Sajai Vir Singh ${ }^{2}$, Dinesh Yadav ${ }^{3}$, Sanjay Kumar Suman ${ }^{4}$, \\ Bhagyalakshmi Lakshminarayanan ${ }^{5}$, Ghanshyam $\operatorname{Singh}^{6}$ \\ ${ }^{1}$ School of Electrical and Electronics Engineering, Fiji National University, Suva, Fiji \\ ${ }^{2}$ Department of Electronics and Communication Engineering, Jaypee Institute of Information Technology, Noida, India \\ ${ }^{3}$ Department of Electronics and Communication Engineering, Manipal University Jaipur, Rajasthan, India \\ ${ }^{4}$ Department Electronics and Communication Engineering, St. Martin's Engineering College, Hyderabad, India \\ ${ }^{5}$ Department Electronics and Communication Engineering, Rajlakshmi Engineering College, Chennai, India \\ ${ }^{6}$ Department of Electronics and Communication Engineering, Malaviya National Institute of Technology Jaipur, Rajasthan, India
}

\begin{abstract}
Article Info
Article history:

Received Nov 18, 2020

Revised Sep 7, 2021

Accepted Oct 2, 2021

\section{Keywords:}

Direction of arrival Interference white noise ratio Minimum power distortion

Minimum variance distortionless response Signal-to-interference and noise ratio

ABSTRACT

This paper introduces a significant special situation where the noise is a collection of D-plane interference signals and the correlated noise of D+1 is less than the number of array components. An optimal beamforming processor based on the minimum variance distortionless response (MVDR) generates and combines appropriate statistics for the D+1 model. Instead of the original space of the $\mathrm{N}$-dimensional problem, the interference signal subspace is reduced to D+1. Typical antenna arrays in many modern communication networks absorb waves generated from multiple point sources. An analytical formula was derived to improve the signal to interference and noise ratio (SINR) obtained from the steering errors of the two beamformers. The proposed MVDR processor-based beamforming does not enforce general constraints. Therefore, it can also be used in systems where the steering vector is compromised by gain. Simulation results show that the output of the proposed beamformer based on the MVDR processor is usually close to the ideal state within a wide range of signal-to-noise ratio and signal-to-interference ratio. The MVDR processor-based beamformer has been experimentally evaluated. The proposed processor-based MVDR system significantly improves performance for large interference white noise ratio (INR) in the sidelobe region and provide an appropriate beam pattern.
\end{abstract}

This is an open access article under the CC BY-SA license.

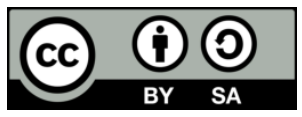

\section{Corresponding Author:}

Satyanand Singh

School of Electrical and Electronics Engineering, Fiji National University

Samabula Fiji Lakeba Street Samabula, Suva, Fiji

Email: Satyanand.singh@fnu.ac.fj

\section{INTRODUCTION}

Beamformers are commonly used in a variety of applications of signal processing, such as voice amplification, radar, and wireless communication. In particular, one of the fundamental problems in array processing is the problem of measuring the signals of several transmitters using an antenna array. The Capon beamformer [1] has gained popularity in this context due to its high ability to refuse interference. Nevertheless, Capon beamformers also known as Van Trees minimum power distortionless response (MPDR) beamformers are still prone to steering errors [2]. Ward, Cox, and Kogon based on diagonal loading effect provides a comparison of several robust MPDR beamformers to correct model errors [3]. Wax et al. [4] performed a theoretical score reading of the signal-to-interference-plus-noise ratio (SINR), which is generalized (that is, not necessarily a diagonal) covariance matrix and the existence of random steering vector 
errors. Interestingly, diagonal loads have proven to be the worst-case solution proposed recently [5]-[7]. The beamformer is built in the latter references to minimize the output power subject to the restriction that the response of the beamformer is above some level for all the steering vectors in an ellipsoid centered around the nominal or presumed value steering vector. In the presence of partially and fully correlated interfering sources, Reddy et al. [8] first study the signal cancelation and interference rejection effects of the optimum (constrained minimum squares or minimum variance) beamformer.

$\mathrm{Gu}$ and Leshem, proposed an effective adaptive beamforming algorithm [9]. This algorithm is robust not only to the covariance matrix uncertainty but also to steering vector mismatch. Using direction of arrival (DOA) knowledge to estimate the covariance of interference and noise that can reconstruct a matrix from a spatial spectral distribution. In the presence of unknown signals, a new robust and adaptive beamforming method was developed by Vorobyov et al. [10] to steer vector mismatch [11]. This method is based on worst-case performance optimization. The natural formulation of this adaptive beamforming problem involves minimizing a quadratic function with an infinite number of non-convex quadratic constraints. The main classification is for a system dedicated to dealing with covariance lattice examples, because apart from the clamor covariance framework, precise obstacles are usually not accessible in near-Earth applications. The best known of this class is called the diagonal loading method [12], [13] where a scaled identity matrix is added to the sample covariance matrix. However, choosing the right diagonal load factor for different situations can be a daunting task. Recently, the shrinkage estimation method [14], [15] automatically measures the diagonal load factor without defining user parameters. Unfortunately, this only provides an approximation of the theoretical received signal covariance matrix, not the required interference plus noise covariance matrix. The second method classifies the signal's expected steering vector explicitly, because it is not easy to obtain accurate steering vector information. In this case, the adaptive beamforming technique based on worst-case performance optimization [16], [17] explicitly uses an uncertainty array of signal control vectors. In fact, the mismatch vectors and their boundary norms are unknown. In this case, the most recent method is to iteratively estimate the actual steering vector [18], [19].

If the array variants are fully known, there is no difference between using the acquired signal covariance matrix MPDR or sample matrix inversion (SMI) and using minimum variance distortionless response (MVDR) [20]. However, if sequence variants are not completely clear, we recommend using the MVDR method [21], [22]. On the other hand, most recently developed robust technologies use a generalization of MPDR technology. Khabbazibasmenj et al. [23], [24] and Mallipeddi et al. [25] recent efforts have been made to isolate the effects of interference. This form of matrix replaces the interference and noise covariance matrices in two published articles. Reference [26] changed the optimal signal control vector to only be used as part of the semi-optimal optimization. This increases the signal loss of the noise output. In [27], the integral is replaced by a discrete DOA, which is determined by minimizing the Capon spectrum estimator. The fact that interference power estimation is not included in the construction of the covariance matrix undermines methods used for high dynamic range applications, such as radio astronomy imaging of diffuse sources. The response is based on the reconstruction of the interference plus noise covariance matrix and the steering vector approximation to evaluate the optimal beamformer for discrete interference [28]. As mentioned above, in order to arrange multiple errors, the MVDR beamformer is more flexible than the MPDR beamformer. New design ideas for multiple-input-multiple-output (MIMO) beamforming antenna arrays for compact and thin handheld devices reported by Liu et al. [29]. A printed array clutch broadband beamforming with four $2.4 \mathrm{GHz}$ multiple input multiple output chip antennas (CA) for 5G communication has been investigated by Hansen et al. [30].

Therefore, under the constraint that the modified steering vector does not converge to interference, the assumed steering vector of the signal is subsequently corrected to optimize the output power of the beamformer. By combining the interference-noising covariance matrix and the adaptive beamformer, a reconstruction based on steering vector estimation can be obtained. These also do not require general restrictions on steering vectors [31], [32].

Therefore, beamforming based on MVDR processors can be extended to more situations than before. Numerical examples show that for low signal-to-noise ratio (SNR) and high SNR performance, using the previously proposed robust beamformer, the performance of the proposed beamforming algorithm is almost always the best [33], [34]. Combining the reconstruction of the interference plus noise covariance matrix with the enhanced estimation of the required signal steering vector can overcome the problem of self-cancellation of the required signal at high SNR, while maintaining good performance at low SNR. This improves performance compared to existing methods and has a wide range of signal-to-interference and noise ratios [35]. In operation, multiple signals from a point source are received by the array. Interference sources and non-coherent sources form a noise correlation matrix. The results show that the MVDR beamformer is still more robust in the presence of uncertain interfering signal steering vectors [36]-[38]. 


\section{SIGNAL COPY AND OPTIMUM DETECTION PROBLEM FORMULATION}

If, as is typically the case, the noise and the desired underlying signal are uncorrelated, then the variance of the reported signal is the sum of the desired signal and noise variances. The MVDR solution is therefore aimed at reducing this number, thereby mitigating the noise effect. A solitary plane wave signal associated to the array manifold $v\left(\omega: K_{S}\right)$ and a signal that interferes with the array manifold $v\left(\omega: K_{1}\right)$, plus the white noise component of individually sensor at the $\sigma_{w}^{2}$ spectral height. Our main goal to find the best undistorted filter, the best typical beamformer array gain, and the best beamformer beam pattern. The block diagram of MDVR processor is shown in Figure 1. A case of plane wave interference reveals several outcomes that can be generalized to many interfering signals.

$$
S_{n}(\omega)=\sigma_{w}^{2} I+M_{1}(w) v\left(\omega: K_{1}\right) v^{H}\left(\omega: K_{1}\right)
$$

In (1) $M_{1}(w)$ is the spectrum of interfering signal $S_{n}=\sigma_{w}^{2} I+M_{1} v_{1} v_{1}^{H}$ can be obtained by suppressing $\omega$ and $K$. Applying the matrix inversion lemma.

$$
S_{n}^{-1}=\frac{1}{\sigma_{w}^{2}}\left[I-\frac{M_{1}}{\sigma_{w}^{2}+N M_{1}} v_{1} v_{1}^{H}\right]
$$

Expected noise at each sensors estimated as follows:

$$
S_{n}=\left(\sigma_{w}^{2}+M_{1}\right)
$$

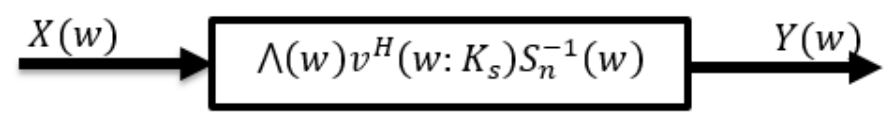

Figure 1. Block diagram of MVDR processor

$$
W_{m v d r}^{H} \stackrel{\Delta}{\rightarrow} W_{o}^{H}(w)=\wedge(w) v^{H}\left(w: K_{s}\right) S_{n}^{-1}(w)
$$

The ratio of the signal to the noise spectrum at the output of the undistorted filter relative to the input ratio gives the array gain at a specific frequency $\omega$. Replace the value of (2). In (4), obtain $W_{m v d r}^{H}$ as shown in (5).

$$
W_{m v d r}^{H}=w_{o}^{H}=\frac{\wedge}{\sigma_{w}^{2}} v_{s}^{H}\left[I-\frac{M_{1}}{\sigma_{w}^{2}+N M_{1}} v_{1} v_{1}^{H}\right]
$$

Defining spatial correlation coefficient $\rho s_{1} \stackrel{\Delta}{\rightarrow} \frac{v_{S}^{H} v_{1}}{N}$ among the anticipated signal to interference ratio. $\rho s_{1}$ is represents predictable beam pattern expected as $K_{S}$ which is the signal wavenumber and estimated at $K_{1}$ is the interfering wave number. Then (5) can be reduce as follows:

$$
W_{0}^{H}=\frac{\wedge N}{\sigma_{w}^{2}}\left[\frac{v_{S}^{H}}{N}-\rho_{S 1} \frac{N M_{1}}{\sigma_{w}^{2}+N M_{1}} \cdot \frac{v_{1}^{H}}{N}\right]
$$

Where, $\wedge$ is the normalizing coefficient given as:

$$
\wedge=\left[\frac{1}{\sigma_{\omega}^{2}} v_{S}^{H}\left\{I-\frac{M_{1}}{\sigma_{\omega}^{2}+N M_{1}} v_{1} v_{1}^{H}\right\} v_{S}\right]^{-1}=\left[\frac{1}{\sigma_{\omega}^{2}} N\left\{1-\frac{N M_{1}}{\sigma_{\omega}^{2}+N M_{1}}\left|\rho_{S 1}\right|^{2}\right\}\right]^{-1}
$$

Optimum MVDR processor of single plane-wave interferer for general and high interference case is shown in Figure 2(a). We see that the beamformer generates two sufficient spatial statistics, one that corresponds to the anticipated signal and one that corresponds interfering signal, and then optimally combines them. Considering that each of the space processors is, respectively, a traditional beamformer pointing to the signal and interferer. Then we deduct a fraction of the estimate that corresponds to the two signals spatial correlation. In case $N M_{1} \gg \sigma_{\omega}^{2}$ then $W_{O}^{H}$ can be derived as [39]: 


$$
W_{0}^{H}=\frac{\wedge}{\sigma_{\omega}^{2}} v_{S}^{H}\left[I-v_{1}\left\{v_{1}^{H} v_{1}\right\}^{-1} v_{1}^{H}\right]=\frac{\wedge}{\sigma_{\omega}^{2}} v_{S}^{H} P \frac{1}{I}
$$

where $P \frac{1}{I}$ is the interference projection matrix on the orthogonal subspace. Figure 2 (b) displays the corresponding device. The beamformer puts on the interfering signal a complete zero. Substituting $S_{n}=\left(\sigma_{w}^{2}+M_{1}\right)$ and $\left[\frac{1}{\sigma_{\omega}^{2}} N\left\{1-\frac{N M_{1}}{\sigma_{\omega}^{2}+N M_{1}}\left|\rho_{S 1}\right|^{2}\right\}\right]^{-1}$ into $A_{0}\left(\omega: k_{S}\right)=S_{n}(\omega) v^{H}\left(\omega: k_{S}\right) S_{n}^{-1}(\omega) v\left(\omega: k_{S}\right)$ to obtain optimum array gain.

$$
A_{0}=N\left(1+\frac{M_{1}}{\sigma_{\omega}^{2}}\right)\left(1-\frac{N M_{1}}{\sigma_{\omega}^{2}+N M_{1}}\left|\rho_{S 1}\right|^{2}\right)
$$

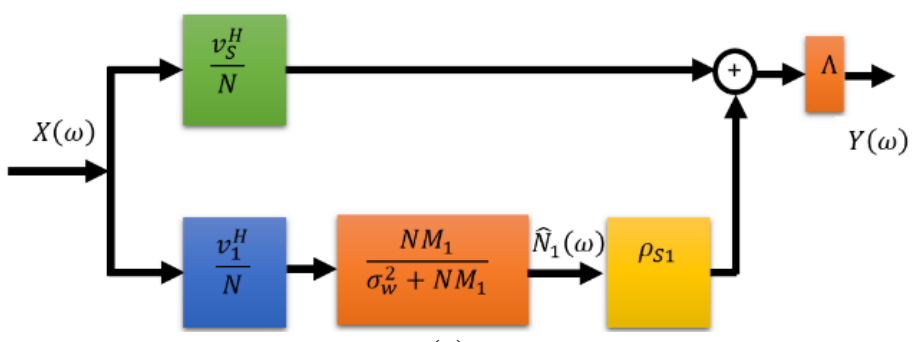

(a)

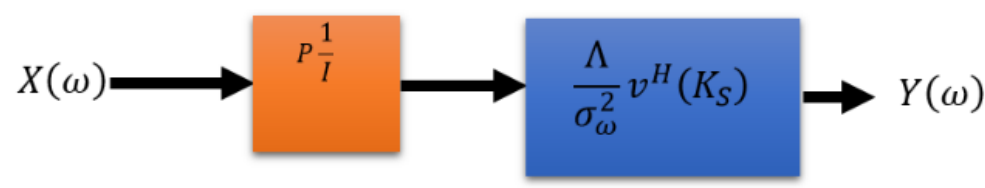

(b)

Figure 2. Optimum MVDR processors: Single plane-wave interferer general and high interference case (a) optimum MVDR processor; single plane-wave interferer general case in correlated signal and interferences and (b) optimum MVDR processor; single plane-wave high interferer

$$
A_{0}=N\left(1+\sigma_{I}^{2}\right)\left[\frac{1+N \sigma_{I}^{2}\left\{1-\left|\rho_{S 1}\right|^{2}\right\}}{1+N \sigma_{I}^{2}}\right]
$$

It is found that the optimal gain of the array depends on $\sigma_{I}^{2}$, INR and $\rho_{S 1}$, that is, the spatial correlation between the signal and interference. Computer simulated first 101 optimum array gain for 10-element array are as follows: 1.009, 11.099, 21.189, 31.279, 41.369, 51.459, 61.548, 71.638, 81.728, $91.818,101.908,111.998,122.088,132.178,142.268,152.358,162.448,172.537,182.627,192.717$, 202.807, 212.897, 222.987, 233.077, 243.167, 253.257, 263.347, 273.437, 283.526, 293.616, 303.706, $313.796,323.886,333.976,344.066,354.156,364.246,374.336,384.426,394.515,404.605,414.695$, $424.785,434.875,444.965,455.055,465.145,475.235,485.325,495.415,505.504,515.594,525.684$, 535.774, 545.864, 555.954, 566.044, 576.134, 586.224, 596.314, 606.404, 616.494, 626.583636.673 646.763

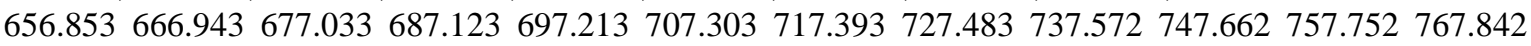

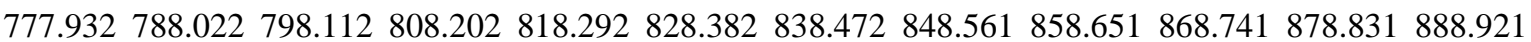
899.011909 .101919 .191929 .281939 .371949 .461959 .550969 .640979 .730989 .820999 .9101010 .000$. Figure 3 represents array gain versus $\left\{1-\left|\rho_{S 1}\right|^{2}\right\}$ : 10 -element array for $I N R=0 d B, I N R=20 d B$ and $I N R=-20 d B$ in correlated signal and interferences. The improvement of the array gain has been observed $24 d B$ for $I N R=20 d B$ and $5.343 d B$ for $I N R=0 d B$ at $\left|\rho_{S 1}\right|^{2}=0.5$ half power beam width (HPBW) line. The improvement of array gain is marginally high for $I N R=20 \mathrm{~dB}$ at $\left|\rho_{S 1}\right|^{2}=0.5$ and $\left|\rho_{S 1}\right|^{2}=0.95$ line compared to $I N R=0 d B$. Using the standard beam pattern $\rho_{S 1}=B_{c}\left(k_{1}, k_{s}\right)$, array geometry must be incorporated in $\rho_{S 1}$ so that these tests are applicable to an arbitrary array. HPBW lines at $\left|\rho_{S 1}\right|^{2}=0.5$ and $\left|\rho_{S 1}\right|^{2}=0.95$ is shown in Figure 3. The interferer is inside the main lobes HPBW region for $\left|\rho_{S 1}\right|^{2}=0.5$ to the left of this axis. Substituting $S_{n}=\sigma_{w}^{2} I+M_{1} v_{1} v_{1}^{H}$ into $A_{c}\left(\omega: k_{s}\right)$ to get the conventional array gain $A_{c}\left(\omega: k_{S}\right)=\frac{N\left(1+\sigma_{I}^{2}\right)}{1+\sigma_{I}^{2} N\left|\rho_{S 1}\right|^{2}}$. 


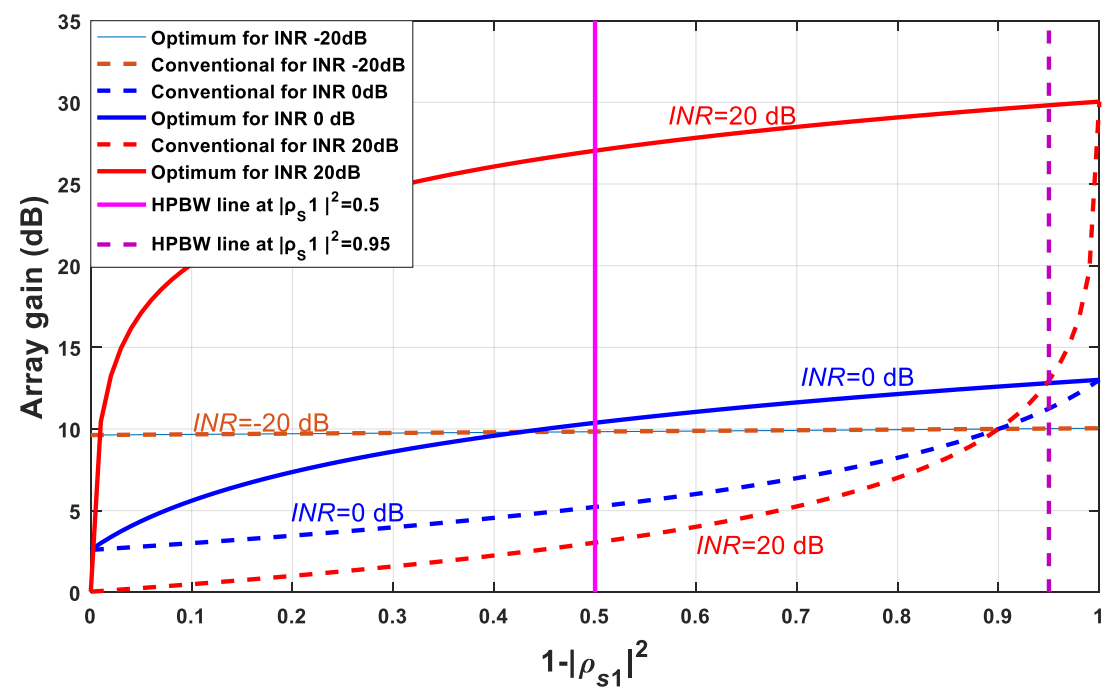

Figure 3. Array gain versus $\left\{1-\left|\rho_{S 1}\right|^{2}\right\}$ : 10-element array: For $=0 d B, I N R=20 d B$ and $I N R=-20 d B$ in correlated signal and interferences

\section{PATTERN PROBLEM ANALYSIS}

Noticed that through the standard beam pattern in the array geometry is encoded in $\rho_{S 1}$ so that these effects are applicable to an arbitrary array. The interferer is inside the main lobes half power beam width (HPBW) region for $\left|\rho_{S 1}\right|^{2}$ to the left of this line. When we look at beam patterns in the examples, we find that this can cause pattern problems. Considering the number of elements $N$ is very large then $N \sigma_{1}^{2}\left[1-\left|\rho_{S 1}\right|^{2}\right] \gg 1$ and optimum array gain $A_{0}$ becomes as in (11).

$$
A_{0} \cong N\left(1+\sigma_{I}^{2}\right)\left(1-\left|\rho_{S 1}\right|^{2}\right)
$$

Computer simulated first 100 optimum array gain for traditional system for 10-element array are as follows: $0.010,0.020,0.030,0.040,0.051,0.061,0.071,0.081,0.091,0.101,0.111,0.121,0.131,0.141$, $0.152,0.162,0.172,0.182,0.192,0.202,0.212,0.222,0.232,0.242,0.253,0.263,0.273,0.283,0.293,0.303$, $0.313,0.323,0.333,0.343,0.354,0.364,0.374,0.384,0.394,0.404,0.414,0.424,0.434,0.444,0.455,0.465$, $0.475,0.485,0.495,0.505,0.515,0.525,0.535,0.545,0.556,0.566,0.576,0.586,0.596,0.606,0.616,0.626$, $\begin{array}{llllllllllllllllll}0.6360 .646 & 0.657 & 0.667 & 0.677 & 0.687 & 0.697 & 0.707 & 0.717 & 0.727 & 0.737 & 0.747 & 0.758 & 0.768 & 0.778 & 0.788 & 0.798\end{array}$

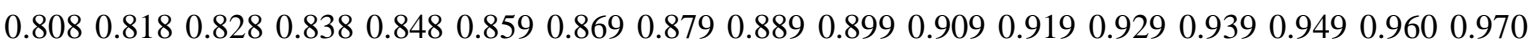
0.9800 .9901 .0001 .010 . Therefore, plotting $A_{0} / N$ as shown in Figure 4(a). The beam pattern can be obtained by substituting $W_{0}^{H}=\frac{\wedge N}{\sigma_{w}^{2}}\left[\frac{v_{s}^{H}}{N}-\rho_{S 1} \frac{N M_{1}}{\sigma_{w}^{2}+N M_{1}} \cdot \frac{v_{1}^{H}}{N}\right]$ in the probability density of $X\left(\omega_{m}\right)$, given $F\left(\omega_{m}\right)$ is for the optimum array represented in mathematical form as follows:

$$
\begin{aligned}
p X\left(\omega_{m}\right) \mid F\left(\omega_{m}\right)(.) & \\
& =c_{1} \exp \left[-\left\{X^{H}\left(\omega_{m}\right)-F^{*}\left(\omega_{m}\right) v^{H}\left(\omega_{m}\right)\right\} S_{n}^{-1}\left(\omega_{m}\right)\left[X\left(\omega_{m}\right)\right.\right. \\
& \left.\left.-F\left(\omega_{m}\right) v\left(\omega_{m}\right)\right]\right]
\end{aligned}
$$

The result is

$$
B_{0}\left(\omega: k_{s}\right)=\frac{\wedge N}{\sigma_{\omega}^{2}}\left[\rho_{s a}-\frac{N \sigma_{I}^{2} \rho_{s 1}}{1+N \sigma_{I}^{2}} \rho_{1 a}\right]
$$

where $\wedge$ is the normalizing coefficient and suppressing the $\omega$ in right side of (13),

$$
\rho_{s a}=\frac{1}{N} v^{H}\left(\omega: k_{s}\right) v(\omega: k)=B_{c}\left(k: k_{s}\right)
$$

which is the traditional beam pattern for a $k_{s}$-pointed delayed and summed of beamformer and that can be expressed mathematically as follows: 


$$
\rho_{1 a}=\frac{1}{N} v^{H}\left(\omega: k_{1}\right) v(\omega: k)=B_{c}\left(k: k_{1}\right)
$$

The (15) is only the typical beam pattern for a $k_{i}$-spot beamformer. Its optimum array pattern can be further interpreted as the traditional beam pattern pointing to the desired signal minus a constant time pointing to the interference by the beam pattern.

$$
B_{0}\left(\omega, k: k_{s}\right)=\frac{\wedge(\omega) \cdot N}{\sigma_{\omega}^{2}} \cdot\left[B_{c}\left(\omega, k: k_{S}\right)-\frac{N \sigma_{I}^{2}}{1+N \sigma_{I}^{2}} \cdot B_{c}\left(k_{1}: k_{s}\right) \cdot B_{c}\left(k: k_{1}\right)\right]
$$

The value of the multiplication factor depends on two elements. The first is the $k_{1}$ value of the standard beam pattern pointing to $k_{s}$. If the interference is zero in legacy mode, it has no effect and does not need to be subtracted. If the source of interference is in the traditional sidelobe, the corresponding sum must be deleted. The second term depends on a value $N \sigma_{I}^{2}$ near unity. The effect of combining these two items is to create partial zeros in the $k_{1}$ beam pattern. The beam pattern value of $k_{1}$ is mathematically expressed as:

$$
B_{0}\left(k_{1}: k_{s}\right)=\frac{\wedge(\omega)}{\sigma_{w}^{2}} \cdot N B_{c}\left(k_{1}: k_{s}\right)\left(\frac{1}{1+N \sigma_{I}^{2}}\right)
$$

As $N \sigma_{I}^{2} \rightarrow \infty$ one can get the optimum array pattern null in the direction of interferer [40].

\section{ANALYSIS OF THE MVDR PROCESSOR BASED BEAMFORMER}

Consider the generic linear array of 10-elements along the $\mathrm{z}$-axis. From $A_{0} \cong N\left(1+\sigma_{I}^{2}\right)$ $\left(1-\left|\rho_{S 1}\right|^{2}\right)$ the standard beam pattern which is designed for an array that is pointed out at broadside $u_{s}=0$ and $u=\cos \theta$ is expressed as $B_{c}(u)$. The region and their range of lobes is shown in Table 1 .

$$
B_{c}(u)=\frac{1}{N} \frac{\sin \left(\frac{N \pi u}{2}\right)}{\sin \left(\frac{\pi u}{2}\right)}
$$

Table 1. Region and their range of lobe

\begin{tabular}{cc}
\hline Region & Range \\
\hline Sidelobe Region & $0.2 \leq|u| \leq 1.0$ \\
Outer Main Lobe & $0.045 \leq|u| \leq 0.2$ \\
HPBW Region & $0<|u|<0.045$ \\
\hline
\end{tabular}

Let us consider that interference is arriving from $u_{I}$ which is shown in Figure 4(b) illustrates the plot of $A_{0}$ as a function of $u_{I} / B W_{N N}, A_{0} / A_{c}$ and $u_{I}$ observed its extreme at the HPBW. It has been observed that the interferer is occurring outside the main lobe that is $u_{I}>0.5 B W_{N N}$ and the optimum array gain is at $N$. INR $d B$. The traditional gain in the array has the similar performance as the $u$-space.

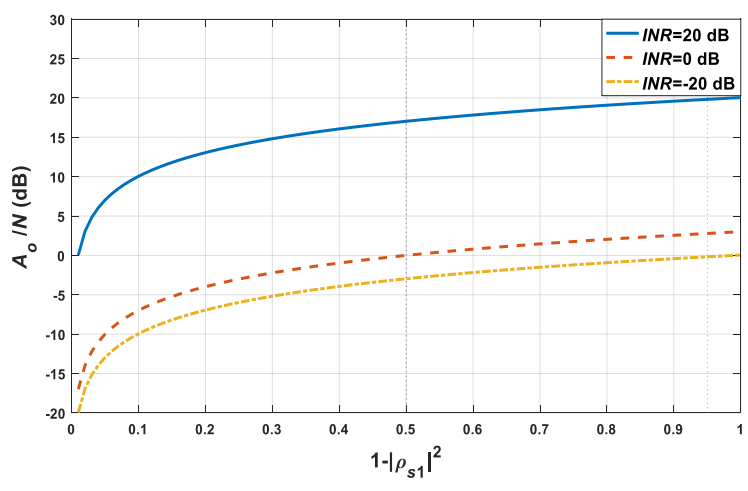

(a)

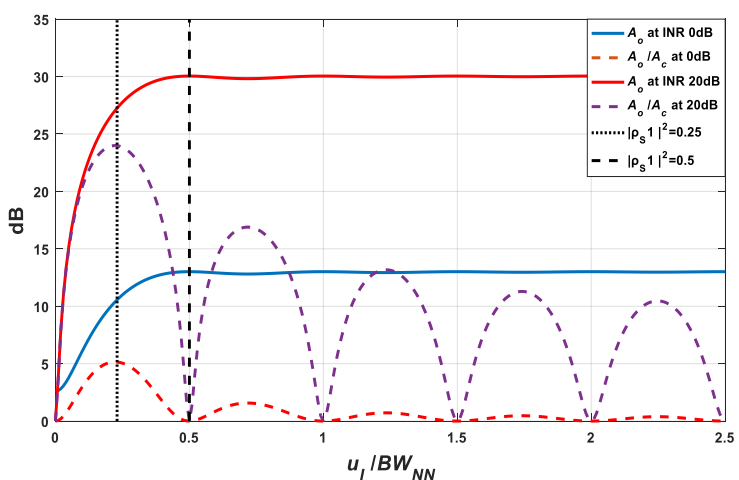

(b)

Figure 4. Correlated signal and interferences normalized optimum array gain (a) Normalized optimum array gain versus $\left\{1-\left|\rho_{S 1}\right|^{2}\right\}$ and (b) $A_{0} / A_{c}$ versus $u_{I} / B W_{N N} ; I N=0 d B$ and $20 \mathrm{dBin}$ 
The best antenna beam patterns of several values of $u_{I}$ in the side lobe region of $10 \mathrm{~dB} \operatorname{IINR}\left(\sigma_{I}^{2}\right)$ are plotted in Figures 5(a) and 5(b). Figures 5(c) and 5(d) demonstration the best antenna beam pattern when the same $u_{I}$ value $\operatorname{INR}\left(\sigma_{I}^{2}\right)$ is $20 \mathrm{~dB}$ in the sidelobe region. It can be seen that the antenna beam pattern is the same as the conventional antenna beam pattern except for the area close to zero.

Figure 6 shows the optimal antenna beam pattern for the main lobe of external interference. Figure 6(a) $\sigma_{I}^{2}=10 d B, u_{I}=0.18$; Figure 6(b) $\sigma_{I}^{2}=10 d B, u_{I}=0.09$; Figure 6(c) $\sigma_{I}^{2}=20 d B, u_{I}=0.18$; Figure 6 (d) $\sigma_{I}^{2}=20 \mathrm{~dB}, u_{I}=0.09$. However, in the case of $u_{I}=0.09$, these two effects are deceptive. (i) The main lobe moves from zero to the left and its height is greater than unity. (ii) The height of the right lobe is approximately $3 \mathrm{~dB}$. Figure 7 illustrates the array gain for different $\sigma_{I}^{2}$ values for $N>20$ as a function of $u_{I} / B W_{N N}$.. Outside the main lobe region, only the square beam pattern is the array gain. Therefore, for $20 \mathrm{~dB}$ $I N R$, performance may be reasonable. However, as the INR increases, the optimal array gain $\left(A_{0}=N\right.$. INR) continues to increase, but the deterministic array gain remains the same.

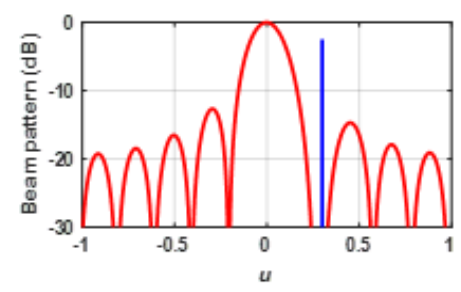

(a)

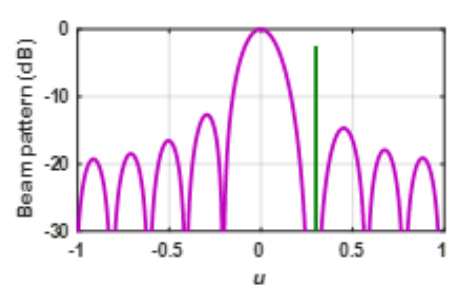

(c)

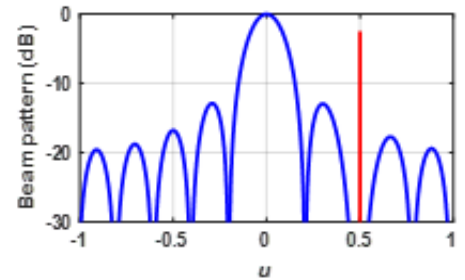

(b)

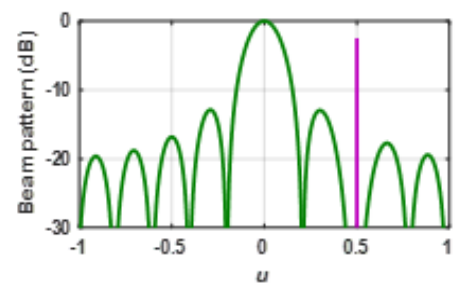

(d)

Figure 5. Optimum antenna beam pattern for side lobe interference (a) $\sigma_{I}^{2}=10 \mathrm{~dB}, u_{I}=0.30$, (b) $\sigma_{I}^{2}=10 \mathrm{~dB}, u_{I}=0.50$, (c) $\sigma_{I}^{2}=20 \mathrm{~dB}, u_{I}=0.30$ and (d) $\sigma_{I}^{2}=20 \mathrm{~dB}, u_{I}=0.50$ in correlated signal and interferences

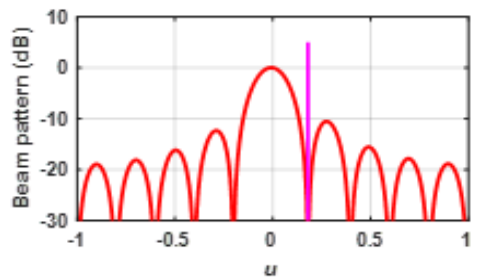

(a)

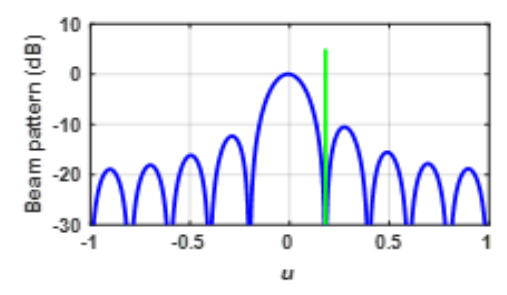

(c)

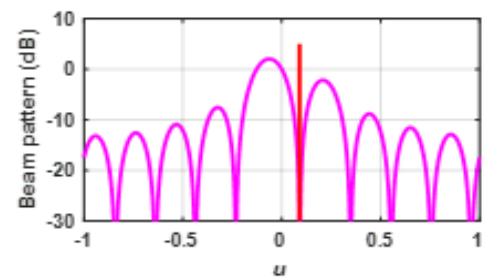

(b)

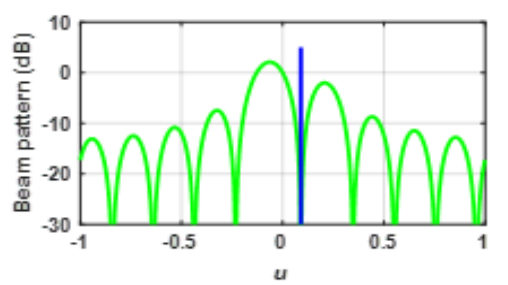

(d)

Figure 6. Optimum antenna beam pattern for outer main-lobe interference (a) $\sigma_{I}^{2}=10 d B, u_{I}=0.18$, (b) $\sigma_{I}^{2}=10 \mathrm{~dB}, u_{I}=0.09$, (c) $\sigma_{I}^{2}=20 \mathrm{~dB}, u_{I}=0.18$ and (d) $\sigma_{I}^{2}=20 \mathrm{~dB}, u_{I}=0.09$ in correlated signal and interferences 


\subsection{White noise plus spatially spread interference consideration}

Let us consider a 31-element standard linear array for MVDR beamformer. We assume that $I N R=20 \mathrm{~dB}, I N R=10 \mathrm{~dB}, I N R=0 \mathrm{~dB} I N R=-10 \mathrm{~dB}$ and $I N R=-20 \mathrm{~dB}$ are five interferers. There are two uncorrelated interfering signals about $\psi=0$ that are symmetrically located. Figure 7 illustrates the array gain for different $\sigma_{I}^{2}$ values for $N>20$ as a function of $u_{I} / B W_{N N}$. In the existence of white noise plus spatially spread interference, reflect the output of MVDR beamformer performance. Considering a regular linear array of 20-elements that is oriented vertically (along the z-axis). The snapshots of the frequency domain can be mathematically represented as:

$$
X(\omega)=v\left(\psi_{t}\right) F(\omega)+N_{c}(\omega)+W(\omega)
$$

The interference comprises a white component with a spectral matrix $\sigma_{\omega}^{2} I$ and a high-surface noise component with a spatial spectral matrix as:

$$
S_{n_{c}}(\omega)=S_{0}(\omega)\left[\operatorname{sinc}\left(k_{0} \Delta p\right)+j \alpha \frac{1}{k_{0} \Delta p}\left\{\operatorname{sinc}\left(k_{0} \Delta p\right)-\cos \left(k_{0} \Delta p\right)\right\} \cos \theta_{p}\right]
$$

in this example, the surface noise is uncorrelated with the white noise. The sensor spacing half wavelength with respect to $\omega c$. The block diagram to obtain the optimum array gain is shown in Figure 8 .

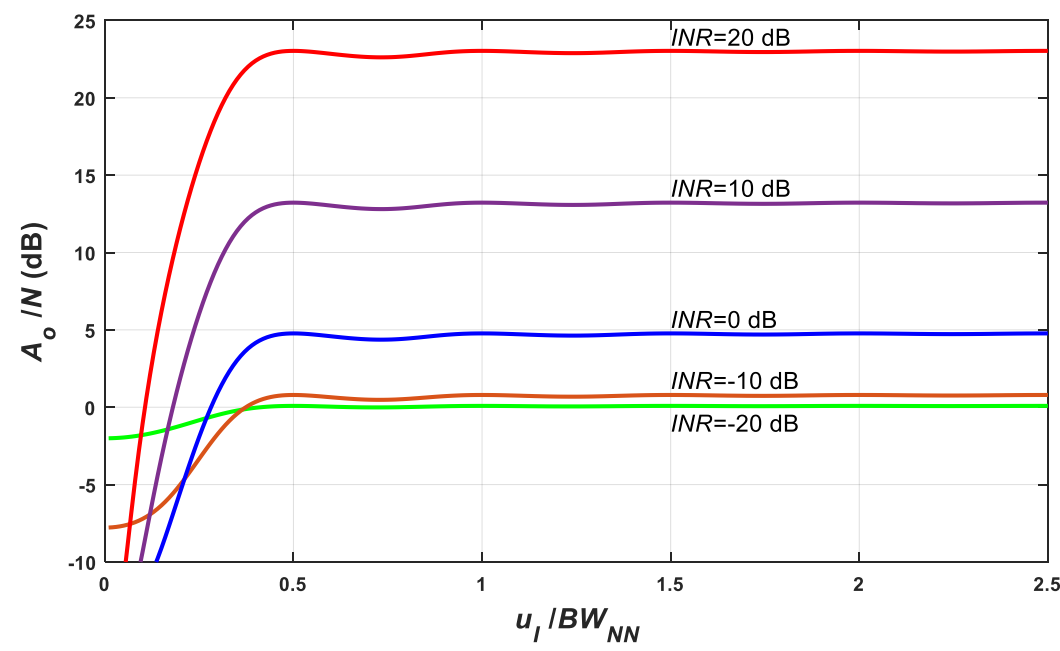

Figure 7. Array gain for different $\sigma_{I}^{2}$ values for $N>20 A_{0} / N(d B)$ as a function of $u_{I} / B W_{N N}$

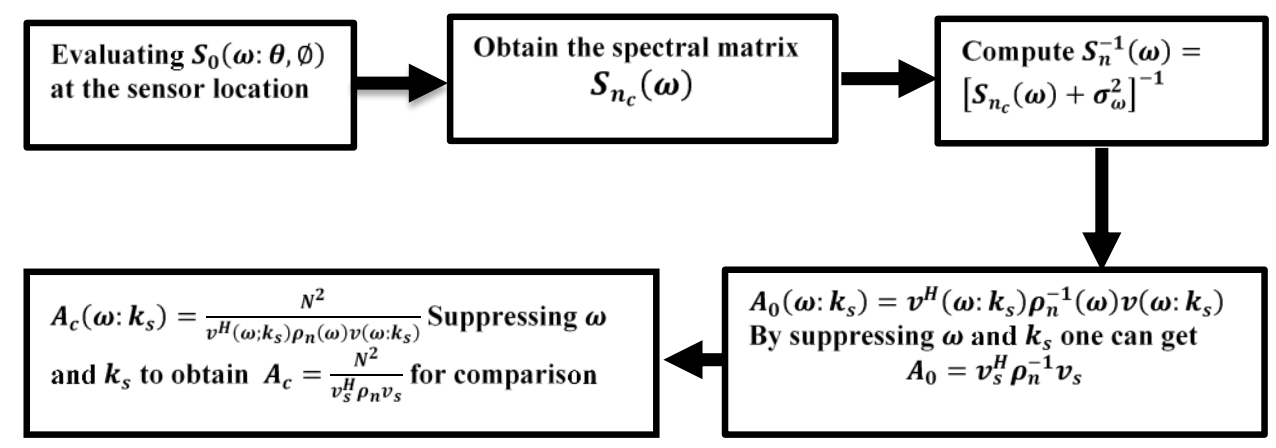

Figure 8. Block diagram to obtain the optimum array gain

Computer generated $10 \times 10$ spectral matrix $S_{n_{c}}(\omega)$ and $S_{n}^{-1}(\omega)=\left[S_{n_{c}}(\omega)+\sigma_{\omega}^{2}\right]^{-1}$ are shown in Table 2 and Table 3 respectively. Computer generated few values of $A_{0}\left(\omega: k_{s}\right)=v^{H}\left(\omega: k_{s}\right) \rho_{n}^{-1}(\omega) v\left(\omega: k_{s}\right)$ 
by suppressing $\omega$ and $k_{s}, A_{0}=v_{s}^{H} \rho_{n}^{-1} v_{s}$ and $A_{c}\left(\omega: k_{s}\right)=\frac{N^{2}}{v^{H}\left(\omega ; k_{s}\right) \rho_{n}(\omega) v\left(\omega: k_{s}\right)}$ by suppressing $\omega$ and $k_{s}$, $A_{c}=\frac{N^{2}}{v_{S}^{H} \rho_{n} v_{s}}$ for comparison is shown in Table 4 .

Table 2. Computer generated $10 \times 10$ spectral matrix $S_{n_{c}}(\omega)$

\begin{tabular}{ccccccccccc}
\hline & $\mathrm{C} 1$ & $\mathrm{C} 2$ & $\mathrm{C} 3$ & $\mathrm{C} 4$ & $\mathrm{C} 5$ & $\mathrm{C} 6$ & $\mathrm{C} 7$ & $\mathrm{C} 8$ & $\mathrm{C} 9$ & $\mathrm{C} 10$ \\
\hline R1 & 101.00 & 10.13 & -2.53 & 1.13 & -0.63 & 0.41 & -0.28 & 0.21 & -0.16 & 0.13 \\
R2 & 10.13 & 101.00 & 10.13 & -2.53 & 1.13 & -0.63 & 0.41 & -0.28 & 0.21 & -0.16 \\
R3 & -2.53 & 10.13 & 101.00 & 10.13 & -2.53 & 1.13 & -0.63 & 0.41 & -0.28 & 0.21 \\
R4 & 1.13 & -2.53 & 10.13 & 101.00 & 10.13 & -2.53 & 1.13 & -0.63 & 0.41 & -0.28 \\
R5 & -0.63 & 1.13 & -2.53 & 10.13 & 101.00 & 10.13 & -2.53 & 1.13 & -0.63 & 0.41 \\
R6 & 0.41 & -0.63 & 1.13 & -2.53 & 10.13 & 101.00 & 10.13 & -2.53 & 1.13 & -0.63 \\
R7 & -0.28 & 0.41 & -0.63 & 1.13 & -2.53 & 10.13 & 101.00 & 10.13 & -2.53 & 1.13 \\
R8 & 0.21 & -0.28 & 0.41 & -0.63 & 1.13 & -2.53 & 10.13 & 101.00 & 10.13 & -2.53 \\
R9 & -0.16 & 0.21 & -0.28 & 0.41 & -0.63 & 1.13 & -2.53 & 10.13 & 101.00 & 10.13 \\
R10 & 0.13 & -0.16 & 0.21 & -0.28 & 0.41 & -0.63 & 1.13 & -2.53 & 10.13 & 101.00 \\
\hline
\end{tabular}

Table 3. Computer generated $10 \times 10$ spectral matrix $S_{n}^{-1}(\omega)=\left[S_{n_{c}}(\omega)+\sigma_{\omega}^{2}\right]^{-1}$

\begin{tabular}{ccccccccccc}
\hline & $\mathrm{C} 1$ & $\mathrm{C} 2$ & $\mathrm{C} 3$ & $\mathrm{C} 4$ & $\mathrm{C} 5$ & $\mathrm{C} 6$ & $\mathrm{C} 7$ & $\mathrm{C} 8$ & $\mathrm{C} 9$ & $\mathrm{C} 10$ \\
\hline R1 & 0.0100 & -0.0011 & 0.0004 & -0.0002 & 0.0001 & -0.0001 & 0.0001 & 0.0000 & 0.0000 & 0.0000 \\
$\mathrm{R} 2$ & -0.0011 & 0.0101 & -0.0011 & 0.0004 & -0.0002 & 0.0001 & -0.0001 & 0.0001 & 0.0000 & 0.0000 \\
R3 & 0.0004 & -0.0011 & 0.0101 & -0.0011 & 0.0004 & -0.0002 & 0.0001 & -0.0001 & 0.0001 & 0.0000 \\
R4 & -0.0002 & 0.0004 & -0.0011 & 0.0101 & -0.0011 & 0.0004 & -0.0002 & 0.0001 & -0.0001 & 0.0001 \\
R5 & 0.0001 & -0.0002 & 0.0004 & -0.0011 & 0.0101 & -0.0011 & 0.0004 & -0.0002 & 0.0001 & -0.0001 \\
R6 & -0.0001 & 0.0001 & -0.0002 & 0.0004 & -0.0011 & 0.0101 & -0.0011 & 0.0004 & -0.0002 & 0.0001 \\
R7 & 0.0001 & -0.0001 & 0.0001 & -0.0002 & 0.0004 & -0.0011 & 0.0101 & -0.0011 & 0.0004 & -0.0002 \\
R8 & 0.0000 & 0.0001 & -0.0001 & 0.0001 & -0.0002 & 0.0004 & -0.0011 & 0.0101 & -0.0011 & 0.0004 \\
R9 & 0.0000 & 0.0000 & 0.0001 & -0.0001 & 0.0001 & -0.0002 & 0.0004 & -0.0011 & 0.0101 & -0.0011 \\
R10 & 0.0000 & 0.0000 & 0.0000 & 0.0001 & 0.0001 & 0.0001 & 0.0002 & 0.0004 & -0.0011 & 0.0100 \\
\hline
\end{tabular}

Table 4. Computer generated few values of $A_{0}=v_{s}^{H} \rho_{n}^{-1} v_{s}$ and $A_{c}=\frac{N^{2}}{v_{S}^{H} \rho_{n} v_{s}}$

\begin{tabular}{cccccccccc}
\hline & Iteration & Iteration & Iteration & Iteration & Iteration & Iteration & Iteration & Iteration & Iteration \\
& 93 & 94 & 95 & 96 & 97 & 98 & 99 & 100 & 101 \\
\hline$A_{0}=v_{s}^{H} \rho_{n}^{-1} v_{s}$ & 78.05 & 66.12 & 56.37 & 48.73 & 42.98 & 38.86 & 36.10 & 34.53 & 34.02 \\
$A_{c}=\frac{N^{2}}{v_{s}^{H} \rho_{n} v_{s}}$ & 42.45 & 34.73 & 29.48 & 25.89 & 23.45 & 21.82 & 20.77 & 20.19 & 20.00 \\
$\theta$ & & & & & & & & \\
& 2.89 & 2.92 & 2.95 & 2.98 & 3.02 & 3.05 & 3.08 & 3.11 & 3.14 \\
\hline
\end{tabular}

Figure 9(a) illustrates high surface noise array gain $(\mathrm{dB})$ vs $\theta_{s}$ for several $S_{n}\left(\omega_{s}\right) / \sigma_{\omega}^{2}$ levels of a MVDR Beamformer. Considering the same model except $S_{n_{c}}(\omega)$ interference comprises a white component with a spectral matrix $\sigma_{\omega}^{2} I$ and a high-layer noise environment with a spatial spectral matrix mathematically represented as in (21):

$$
\begin{aligned}
S_{n_{c}}(\omega)=S_{0}(\omega)[ & \operatorname{sinc}\left(k_{0} \Delta p\right) \\
& +\alpha\left\{\left(\frac{3}{\left(k_{0} \Delta p\right)^{2}}-1\right) \operatorname{sinc}\left(k_{0} \Delta p\right)\right. \\
& \left.\left.-3 \cos \left(k_{0} \Delta p\right)\right\}\left\{\frac{3 \cos \left(2 \theta_{p}\right)+1}{4}\right\}\right]
\end{aligned}
$$

To get the optimal array gain and the traditional array gain, we repeat the same steps. Figure 9(b) illustrates high-level noise environment array gain (dB) vs $\theta_{s}$ for several $S_{n}\left(\omega_{s}\right) / \sigma_{\omega}^{2}$ levels of a MVDR Beamformer [39], [40].

In terms of hardware resources, speed (weight updating rate), algorithms implemented and applications proposed, the present work is compared with earlier recorded works [41], [42]. Table 5 shows the comparison. Although the proposed MVDR beamformer has high array gain for high surface noise compared to conventional. Comparing the results of the proposed study is not a reasonable estimate because 
the literature research work was performed on multiple technologies (FPGA) and platforms, but the authors work are based on higher surface noise array gains than in the previous work [43]. The proposed work mainly focuses on improving the array gain throughput. According to the author's knowledge, this is the first reported processor based MVDR architecture that enables real-time implementation of MVDR beamformer to achieve high array gain with high surface noise. Compared to the latest technology, it is novel in that it has the best array gain for high surface noise and high-level noise throughput.

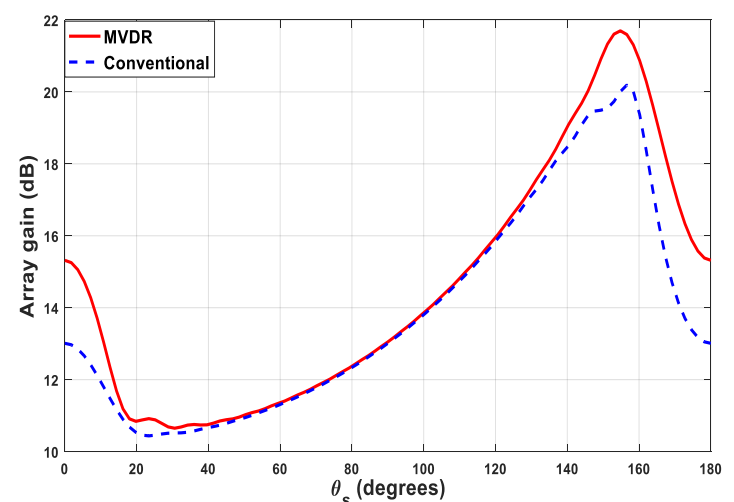

(a)

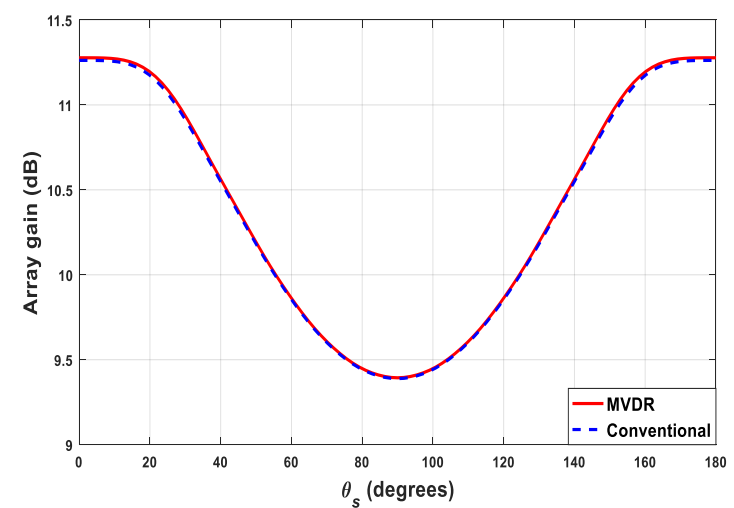

(b)

Figure 9. Array gain (dB) vs $\theta_{s}$ for several $S_{n}\left(\omega_{s}\right) / \sigma_{\omega}^{2}$ levels of a MVDR: (a) beamformer for high surface noise and (b) beamformer for high layer noise

Table 5. Performance comparison of MVDR with previous literature works

\begin{tabular}{|c|c|c|c|c|c|c|}
\hline Scheme & $\begin{array}{c}\text { No of } \\
\text { channels/Matrix } \\
\text { size }\end{array}$ & $\begin{array}{c}\text { Adaptive weight } \\
\text { computation (AWC) } \\
\text { /Matrix inversion (MI) }\end{array}$ & $\begin{array}{l}\text { Updating Rate } \\
\text { (K/Sec) }\end{array}$ & $\begin{array}{l}\text { Clock } \\
\text { cycles }\end{array}$ & $\begin{array}{l}\text { Array } \\
\text { Gain in } \\
\text { dB }\end{array}$ & Algorithm \\
\hline Proposed & $10 \times 10$ & MI & 120.5 & 1600 & 21.7 & Processor based \\
\hline Kidav et al. [44] & 32 & AWC & 132.2 & 1600 & - & Modified complex \\
\hline Ma et al. [45] & $4 \times 4$ & MI & $\begin{array}{c}17.62 \\
\text { MInv/Sec }\end{array}$ & - & - & $\begin{array}{l}\text { Modified squared } \\
\text { givens rotation }\end{array}$ \\
\hline Rosado et al. [46] & $23 \times 23$ & MI & 3.9 & 3441 & - & $\begin{array}{l}\text { Qrd, modified gram- } \\
\text { schmidt method }\end{array}$ \\
\hline Wang et al. [47] & $23 \times 23$ & MI & - & - & 20.0 & $\begin{array}{c}\text { Maximum Diffuse } \\
\text { noise gain beamformer }\end{array}$ \\
\hline
\end{tabular}

\section{CONCLUSION}

To this end, we propose an efficient optimal beamformer for discrete interference. It is robust not only to the volatility of the covariance matrix but also to vector steering. Based on the spatial spectrum distribution of the quasi-signal free environment, the interference plus noise covariance matrix can be reconstructed with the estimated DOA of the signal of interest. Based on the reconstructed covariance matrix, the estimated steering vector of the signal can be modified to optimize the output power of the array. Unlike other algorithms, the proposed optimal beamformer for discrete interference does not require standard steering vector constraints, thus greatly expanding its coverage. Simulation results show that the performance of the proposed optimal beamformer for discrete interference provides a significant performance improvement for large INRs in the sidelobe region and provides an acceptable beam pattern. MVDR beamformer produces solutions that distort the main lobe of the HPBW region and are prone to mismatch modeling. Considering multiple perturbation effects with numerous factors and enhancing MVDR array benefit for complex scenario processing, which is our future research work.

\section{REFERENCES}

[1] J. Capon, "High-resolution frequency-wavenumber spectrum analysis," Proceedings of the IEEE, vol. 57, no. 8, pp. 1408-1418, 1969, doi: 10.1109/PROC.1969.7278.

[2] A. P. Melikhova and I. A. Tsikin, "Optimum array processing with unknown attitude parameters for GNSS anti-spoofing integrity monitoring," in 201841 st International Conference on Telecommunications and Signal Processing (TSP), Jul. 2018, pp. 1-4, doi: 10.1109/TSP.2018.8441358.

[3] J. Ward, H. Cox, and S. M. Kogon, "A comparison of robust adaptive beamforming algorithms," in The Thrity-Seventh Asilomar 
Conference on Signals, Systems and Computers, 2003, pp. 1340-1344, doi: 10.1109/ACSSC.2003.1292206.

[4] M. Wax and Y. Anu, "Performance analysis of the minimum variance beamformer in the presence of steering vector errors," IEEE Transactions on Signal Processing, vol. 44, no. 4, pp. 938-947, Apr. 1996, doi: 10.1109/78.492546.

[5] Jian Li, P. Stoica, and Zhisong Wang, "On robust Capon beamforming and diagonal loading," in 2003 IEEE International Conference on Acoustics, Speech, and Signal Processing, 2003. Proceedings. (ICASSP '03)., vol. 5, pp. V-337-40, doi: 10.1109/ICASSP.2003.1199947.

[6] Z. Zheng, T. Yang, W.-Q. Wang, and S. Zhang, "Robust adaptive beamforming via coprime coarray interpolation," Signal Processing, vol. 169, Art. no. 107382, Apr. 2020, doi: 10.1016/j.sigpro.2019.107382.

[7] R. G. Lorenz and S. P. Boyd, "Robust minimum variance beamforming," IEEE Transactions on Signal Processing, vol. 53, no. 5, pp. 1684-1696, May 2005, doi: 10.1109/TSP.2005.845436.

[8] V. Reddy, A. Paulraj, and T. Kailath, "Performance analysis of the optimum beamformer in the presence of correlated sources and its behavior under spatial smoothing," IEEE Transactions on Acoustics, Speech, and Signal Processing, vol. 35, no. 7, pp. 927-936, Jul. 1987, doi: 10.1109/TASSP.1987.1165239.

[9] Yujie Gu and A. Leshem, "Robust adaptive beamforming based on interference covariance matrix reconstruction and steering vector estimation," IEEE Transactions on Signal Processing, vol. 60, no. 7, pp. 3881-3885, Jul. 2012, doi: 10.1109/TSP.2012.2194289.

[10] S. A. Vorobyov, A. B. Gershman, and Z.-Q. Luo, "Robust adaptive beamforming using worst-case performance optimization: a solution to the signal mismatch problem," IEEE Transactions on Signal Processing, vol. 51, no. 2, pp. 313-324, Feb. 2003, doi: 10.1109/TSP.2002.806865.

[11] S. F. Shaukat, M. ul Hassan, R. Farooq, H. U. Saeed, and Z. Saleem, "Sequential studies of beamforming algorithms for smart antenna systems," World Applied Sciences Journal, vol. 6, no. 6, pp. 754-758, 2009.

[12] H. Cox, R. Zeskind, and M. Owen, "Robust adaptive beamforming," IEEE Transactions on Acoustics, Speech, and Signal Processing, vol. 35, no. 10, pp. 1365-1376, Oct. 1987, doi: 10.1109/TASSP.1987.1165054.

[13] Y. Yang, B. Zheng, S. Zhang, and R. Zhang, "Intelligent reflecting surface meets OFDM: protocol design and rate maximization," IEEE Transactions on Communications, vol. 68, no. 7, pp. 4522-4535, Jul. 2020, doi: 10.1109/TCOMM.2020.2981458.

[14] B. Zheng and R. Zhang, "Intelligent reflecting surface-enhanced OFDM: channel estimation and reflection optimization," IEEE Wireless Communications Letters, vol. 9, no. 4, pp. 518-522, 2020, doi: 10.1109/LWC.2019.2961357.

[15] P. Stoica, Jian Li, Xumin Zhu, and J. R. Guerci, "On using a priori knowledge in space-time adaptive processing," IEEE Transactions on Signal Processing, vol. 56, no. 6, pp. 2598-2602, Jun. 2008, doi: 10.1109/TSP.2007.914347.

[16] P. Saxena and A. G. Kothari, "Performance analysis of adaptive beamforming algorithms for smart antennas," IERI Procedia, vol. 10, pp. 131-137, 2014, doi: 10.1016/j.ieri.2014.09.101.

[17] W. Tang et al., "MIMO transmission through reconfigurable intelligent surface: system design, analysis, and implementation," IEEE Journal on Selected Areas in Communications, vol. 38, no. 11, pp. 2683-2699, 2020, doi: 10.1109/JSAC.2020.3007055.

[18] A. Hassanien, S. A. Vorobyov, and K. M. Wong, "Robust adaptive beamforming using sequential quadratic programming: An iterative solution to the mismatch problem," IEEE Signal Processing Letters, vol. 15, pp. 733-736, 2008, doi: 10.1109/LSP.2008.2001115.

[19] H. A. Leftah and H. N. Alminshid, "Channel capacity and performance evaluation of precoded MIMO-OFDM system with largesize constellation," International Journal of Electrical and Computer Engineering (IJECE), vol. 9, no. 6, pp. 5024-5030, Dec. 2019, doi: 10.11591/ijece.v9i6.pp5024-5030.

[20] Y.-J. Gu, Z.-G. Shi, K. S. Chen, and Y. Li, "Robust adaptive beamforming for steering vector uncertainties based on equivalent doas method," Progress In Electromagnetics Research, vol. 79, pp. 277-290, 2008, doi: 10.2528/PIER07102202.

[21] L. Ehrenberg, S. Gannot, A. Leshem, and E. Zehavi, "Sensitivity analysis of MVDR and MPDR beamformers," in 2010 IEEE 26th Convention of Electrical and Electronics Engineers in Israel, Nov. 2010, pp. 000416-000420, doi: 10.1109/EEEI.2010.5662190.

[22] A. H. Majeed and K. H. Sayidmarie, "UWB elliptical patch monopole antenna with dual-band notched characteristics," International Journal of Electrical and Computer Engineering (IJECE), vol. 9, no. 5, pp. 3591-3598, Oct. 2019, doi: 10.11591/ijece.v9i5.pp3591-3598.

[23] M. O. Al-Dwairi, "A planar UWB semicircular-shaped monopole antenna with quadruple band notch for WiMAX, ARN, WLAN, and X-Band," International Journal of Electrical and Computer Engineering (IJECE), vol. 10, no. 1, pp. 908-918, Feb. 2020, doi: 10.11591/ijece.v10i1.pp908-918.

[24] A. Khabbazibasmenj, S. A. Vorobyov, and A. Hassanien, "Robust adaptive beamforming via estimating steering vector based on semidefinite relaxation," in 2010 Conference Record of the Forty Fourth Asilomar Conference on Signals, Systems and Computers, Nov. 2010, pp. 1102-1106, doi: 10.1109/ACSSC.2010.5757574.

[25] R. Mallipeddi, J. P. Lie, S. G. Razul, P. N. Suganthan, and C. M. S. See, "Robust adaptive beamforming based on covariance matrix reconstruction for look direction mismatch," Progress In Electromagnetics Research Letters, vol. 25, pp. 37-46, 2011, doi: 10.2528/PIERL11040104.

[26] S. Mohammadzadeh and O. Kukrer, "Adaptive beamforming based on theoretical interference-plus-noise covariance and direction-of-arrival estimation,” IET Signal Processing, vol. 12, no. 7, pp. 819-825, Sep. 2018, doi: 10.1049/iet-spr.2017.0462.

[27] T. Li and W. Geyi, "Design of MIMO beamforming antenna array for mobile handsets," Progress In Electromagnetics Research $C$, vol. 94, pp. 13-28, 2019, doi: 10.2528/PIERC19030807.

[28] C. Lee, M. K. Khattak, and S. Kahng, "Wideband 5G beamforming printed array clutched by LTE-A 4×4-multiple-inputmultiple-output antennas with high isolation," IET Microwaves, Antennas \& Propagation, vol. 12, no. 8, pp. 1407-1413, Jul. 2018, doi: 10.1049/iet-map.2017.0946.

[29] J. Liu, W. Liu, H. Liu, Z.-J. Zhang, and B. Chen, "Performance of the SMI beamformer with signal steering vector errors in heterogeneous environments," Signal Processing, vol. 123, pp. 22-29, Jun. 2016, doi: 10.1016/j.sigpro.2015.12.011.

[30] C. K. Hansen, K. F. Warnick, B. D. Jeffs, J. R. Fisher, and R. Bradley, "Interference mitigation using a focal plane array," Radio Science, vol. 40, no. 5, p. n/a-n/a, Oct. 2005, doi: 10.1029/2004RS003138.

[31] A. Farhood, M. K. Naji, S. H. R. H. Rhaif, and A. Ali, "Design and analysis of dual band integrated hexagonal shaped microstrip UWB antenna," Indonesian Journal of Electrical Engineering and Computer Science (IJEECS), vol. 15, no. 1, pp. 294-299, Jul. 2019, doi: 10.11591/ijeecs.v15.i1.pp294-299.

[32] R. P. Labade, S. B Deosarkar, and N. Pisharoty, "Compact integrated Bluetooth UWB antenna with quadruple bandnotched characteristics," International Journal of Electrical and Computer Engineering (IJECE), vol. 5, no. 6, pp. 1433-1440, Dec. 2015, doi: 10.11591/ijece.v5i6.pp1433-1440.

[33] P. P. D and E. N. V, "Mobile tower radiation and its impacts on child health: a study conducted in an ecologically sensitive area

Int J Elec \& Comp Eng, Vol. 12, No. 2, April 2022: 1732-1743 
of western ghats," International Journal of Electrical and Computer Engineering (IJECE), vol. 8, no. 6, pp. 4432-4437, Dec. 2018, doi: 10.11591/ijece.v8i6.pp4432-4437.

[34] S. R. A. Mutalik, M. Hafizuddin Mat, M. Jusoh, and A. W. N. Husna, "A Study of specific absorption rate in human head due to electromagnetic exposure to 4G signals," Indonesian Journal of Electrical Engineering and Computer Science (IJEECS), vol. 13, no. 3, pp. 1161-1166, Mar. 2019, doi: 10.11591/ijeecs.v13.i3.pp1161-1166.

[35] Y. S. Alwan, M. S. Zidan, and M. Q. Taha, "Evaluation of mobile microwave radiation severity at al-door residential complex in Iraq," Indonesian Journal of Electrical Engineering and Computer Science (IJEECS), vol. 14, no. 3, pp. 1281-1285, Jun. 2019, doi: 10.11591/ijeecs.v14.i3.pp1281-1285.

[36] A. N and U. S V, "Obstacle avoidance and distance measurement for unmanned aerial vehicles using monocular vision," International Journal of Electrical and Computer Engineering (IJECE), vol. 9, no. 5, pp. 3504-3511, Oct. 2019, doi: 10.11591/ijece.v9i5.pp3504-3511.

[37] S. Kumar Debnath et al., "A review on graph search algorithms for optimal energy efficient path planning for an unmanned air vehicle," Indonesian Journal of Electrical Engineering and Computer Science (IJEECS), vol. 15, no. 2, pp. 743-749, Aug. 2019, doi: 10.11591/ijeecs.v15.i2.pp743-749.

[38] L. C. Godara, "Applications of antenna arrays to mobile communications. I. Performance improvement, feasibility, and system considerations," Proceedings of the IEEE, vol. 85, no. 7, pp. 1031-1060, Jul. 1997, doi: 10.1109/5.611108.

[39] N. A. Malek, O. O. Khalifa, Z. Z. Abidin, S. Y. Mohamad, and N. A. A. Rahman, "Beam steering using the active element pattern of antenna array," TELKOMNIKA (Telecommunication Computing Electronics and Control), vol. 16, no. 4, pp. 1542-1550, Jun. 2018, doi: 10.12928/telkomnika.v16i4.9040.

[40] M. W. Sabri, N. A. Murad, and M. K. A. Rahim, "Bi-directional beams waveguide slotted antenna at millimeter wave," TELKOMNIKA (Telecommunication Computing Electronics and Control), vol. 16, no. 4, pp. 1515-1521, Aug. 2018, doi: 10.12928/telkomnika.v16i4.9057.

[41] I. H. Idris, M. R. Hamid, K. Kamardin, and M. K. A. Rahim, "Wide to multiband elliptical monopole reconfigurable antenna for multimode systems applications," TELKOMNIKA (Telecommunication Computing Electronics and Control), vol. 17, no. 4, pp. 1663-1669, Aug. 2019, doi: 10.12928/telkomnika.v17i4.12764.

[42] R. H. Thaher and N. B. Hassan, "Proposed p-shaped microstrip antenna array for wireless communication applications," TELKOMNIKA (Telecommunication Computing Electronics and Control), vol. 16, no. 6, Art. no. 2597, Dec. 2018, doi: 10.12928/telkomnika.v16i6.10494.

[43] S. Elajoumi, A. Tajmouati, J. Zbitou, A. Errkik, A. M. Sanchez, and M. Latrach, "Bandwidth enhancement of compact microstrip rectangular antennas for UWB applications," TELKOMNIKA (Telecommunication Computing Electronics and Control), vol. 17, no. 3, pp. 1559-1568, Jun. 2019, doi: 10.12928/telkomnika.v17i3.9184.

[44] J. U. Kidav, N. M. Siva Mangai, and M. P. Pillai, "Design of AWC core using DCD iterations for MVDR beamformer," Microprocessors and Microsystems, vol. 73, no. 1, Art. no. 102969, Mar. 2020, doi: 10.1016/j.micpro.2019.102969.

[45] L. Ma, K. Dickson, J. McAllister, and J. McCanny, "QR decomposition-based matrix inversion for high performance embedded MIMO receivers," IEEE Transactions on Signal Processing, vol. 59, no. 4, pp. 1858-1867, Apr. 2011, doi: 10.1109/TSP.2011.2105485

[46] A. Rosado, T. Iakymchuk, M. Bataller, and M. Wegrzyn, "Hardware-efficient matrix inversion algorithm for complex adaptive systems," in 2012 19th IEEE International Conference on Electronics, Circuits, and Systems (ICECS 2012), Dec. 2012, pp. 41-44, doi: 10.1109/ICECS.2012.6463562.

[47] X. Wang, I. Cohen, J. Chen, and J. Benesty, "On robust and high directive beamforming with small-spacing microphone arrays for scattered sources," IEEE/ACM Transactions on Audio, Speech, and Language Processing, vol. 27, no. 4, pp. 842-852, Apr. 2019, doi: 10.1109/TASLP.2019.2899517. 